\title{
Cas proteins: dodgy scaffolding in breast cancer
}

\author{
Giusy Tornillo ${ }^{1,2}$, Paola Defilippi ${ }^{1}$ and Sara Cabodi ${ }^{{ }^{*}}$
}

\begin{abstract}
The members of the Cas protein family (p130Cas/ BCAR1, Nedd9/HEF1, EFS and CASS4) are scaffold proteins required for the assembly of signal transduction complexes in response to several stimuli, such as growth factors, hormones and extracellular matrix components. Given their ability to integrate and coordinate multiple signalling events, Cas proteins have emerged as crucial players in the control of mammary cell proliferation, survival and differentiation. More importantly, it has been found that alterations of their expression levels result in aberrant signalling cascades, which promote initiation and progression of breast cancer. Based on the increasing data from in vitro, mouse model and clinical studies, in this review we will focus on two Cas proteins, p130Cas/BCAR1 and Nedd9, and their coupled signalling pathways, to examine their role in mammary cell transformation and in the acquirement of invasiveness and drug resistance of breast cancer cells.
\end{abstract}

\section{Introduction}

Mammary gland development is the result of coordinated actions of hormones, growth factors and extracellular matrix signalling pathways. Not surprisingly, deregulation of proteins acting at various points within these pathways can contribute to the onset and progression of breast cancer. Cas proteins serve as scaffolds to regulate the assembly of intracellular signalling complexes downstream of numerous receptors, such as growth factor and hormone receptors as well as integrins. Thus, they play a key role in integrating multiple signalling networks, which together ultimately govern cell survival, proliferation and migration [1].

The Cas family comprises four members: p130Cas/ BCAR1 (p130 Crk-associated substrate; also known as Breast cancer anti-estrogen resistance 1 (BCAR1)), Nedd9

\footnotetext{
* Correspondence: sara.cabodi@unito.it

'Department of Molecular Biotechnology and Health Sciences, University of Torino, Torino 10126, Italy

Full list of author information is available at the end of the article
}

(Neural precursor cell expressed, developmentally downregulated 9; also called Human enhancer of filamentation 1 (HEF-1) or Cas-L), EFS (Embryonal Fyn-associated substrate) and CASS4 (Cas scaffolding protein family member 4). These proteins have a similar structure, which is characterized by the presence of multiple protein interaction domains and several tyrosine and serine phosphorylation motifs [2] (Figure 1).

Over the past decade, p130Cas/BCAR1 and Nedd9 have emerged as key players in the control of many different aspects of mammary gland biology, including mammary epithelial cell homeostasis and mammary tumour cell behaviour. More interestingly, despite their high similarity, they regulate these processes through both distinct and overlapping mechanisms.

In this review, we summarize and discuss the most recent findings regarding the role of p130Cas/BCAR1 and Nedd9 in the normal mammary epithelium and in different breast cancer subtypes.

\section{p130Cas/BCAR1 and Nedd9 in the normal mammary epithelium}

Several studies have shown that, in normal human breast tissue, both p130Cas/BCAR1 and Nedd9 are mainly present in the mammary epithelium rather than in stromal cells, although at relatively low levels and with a heterogeneous expression pattern [3-6]. Even if detected in all the distinct epithelial cell populations, p130Cas/BCAR1 expression is highly enriched in the basal compartment of the mouse mammary epithelium [4]. Similarly, Nedd9 is almost exclusively expressed in basal cells in the normal human mammary epithelium [7]. During late pregnancy and lactation p130Cas/BCAR1 is down-regulated [4], whereas the expression of Nedd9 has never been investigated at different stages of mammary gland development.

The regulation of p130Cas/BCAR1 levels is crucial for proper mammary morphogenesis and tissue homeostasis. Over-expression of p130Cas/BCAR1 enhances mammary branching morphogenesis in vivo during puberty as well as ex vivo upon stimulation with either epidermal growth factor or fibroblast growth factor $[3,8]$. Moreover, in threedimensional organotypic cultures of NmuMg mammary 


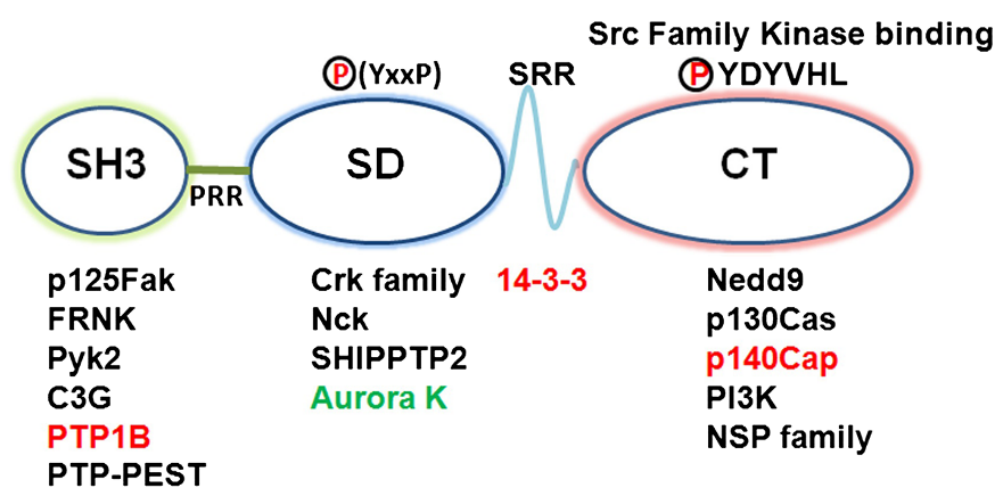

Figure 1 p130Cas/BCAR1 and Nedd9 structural features and main interacting proteins. Schematic representation of Cas protein main domains: an amino-terminal SH3 domain followed by a proline-rich region (PRR), a substrate domain (SD), a serine rich region (SRR), and a carboxy-terminal domain (CT). The main proteins whose interaction has been demonstrated for both p130Cas and Nedd9 are indicated in black, the interactors specific for p130Cas are in red and those specific for Nedd9 are in green. Noteworthy, p125FAK binds to the SH3 domain while the Src family kinases bind to the CT domain. The substrate domain with the multiple YxxP motifs represents the site where Src family kinases extensively phosphorylate Cas proteins.

cells, high levels of p130Cas/BCAR1 elicit the formation of acini with a filled lumen [9]. Similar defects in lumen clearance can be evoked in primary mouse mammary explants when p130Cas/BCAR1 over-expression is specifically combined with epidermal growth factor and oestrogen treatment [8]. These indications for p130Cas having a role as a positive regulator of mammary cell growth are strengthened by the fact that in vivo over-expression of p130Cas/BCAR1 causes extensive hyperplasia throughout mammary gland development, delayed involution at the end of lactation, along with persistent proliferation, decreased apoptosis and hyper-activation of Src, Erk1/2 and Akt signalling pathways [3].

A likely explanation for the hyper-proliferative phenotype associated with high p130Cas/BCAR1 levels might be the expansion of the progenitor cell population that occurs following p130Cas/BCAR1 over-expression [4]. Up-regulation of p130Cas also impairs the ability of mammary luminal progenitors to differentiate in response to lactogenic stimuli and shifts their commitment towards the basal cell fate [4]. Remarkably, these alterations in the mammary progenitor cell behaviour depend on the abnormal activation of the c-Kit tyrosine kinase receptor, thus revealing a novel function of this receptor in the control of mammary cell differentiation [4].

The consequences of p130Cas/BCAR1 depletion in vivo in the mammary epithelium remain to be understood. Transgenic mice expressing the constitutively phosphorylated substrate domain (SD) of p130Cas/BCAR1 (MMTV$\mathrm{Src}^{*} / \mathrm{SD}$ p130Cas), which has been reported to act as a p130Cas/BCAR1 dominant-negative mutant in vitro, do not exhibit any gross morphological alterations of the mammary gland [10]. However, the Src*/SD p130Cas mutant does not appear to effectively inhibit endogenous p130Cas/BCAR1 in vivo. Nevertheless, previous studies suggest that p130Cas/BCAR1 is required for mammary epithelial cells to survive and grow in vitro [9].

Lack of Nedd9 does not significantly affect mammary gland development [11], but whether p130Cas/BCAR1 might be able to compensate for Nedd9 deficiency remains to be investigated. Yet, more recently, it has been shown that in NEDD9-null mammary glands, the frequency of luminal progenitors is reduced, while the basal epithelial cell population is not altered [12]. However, although NEDD9-null mammary luminal progenitors show altered expression of genes involved in mitosis, replicative stress and centrosome maturation, no defect in their ability to grow in vitro has been observed. This finding, together with the selective expression of Nedd9 in basal cells reported by Bruna and colleagues [7], suggests that the effects of Nedd9 depletion in the luminal cell pool in vivo may be non-cell autonomous effects.

\section{p130Cas/BCAR1 and Nedd9 signalling in breast cancer: from cell transformation to tumour cell dissemination}

The relevance of the adaptor proteins p130Cas/BCAR1 and Nedd 9 in breast cancer has been highlighted by numerous in vivo and in vitro studies. Both p130Cas/BCAR1 and Nedd9 are abundantly expressed in human breast tumours and breast cancer cell lines $[3,5,6,13]$.

In particular, p130Cas/BCAR1 protein expression has been found markedly increased in a large subset of human breast tumours but with no correlation with tumour size or lymph node status. In spite of this, in patients with primary breast cancer, high BCAR1/p130Cas levels are associated with poor relapse-free survival and poor overall survival $[13,14]$. Moreover, up-regulation of p130Cas/BCAR1 in benign breast lesions indicates that its over-expression may occur early during mammary 
tumourigenesis [3]. Like p130Cas/BCAR1, Nedd9 is strongly over-expressed in human breast cancers with respect to normal tissue. Specifically, Nedd9 expression positively correlates with a series of adverse prognostic markers, including high tumour grade [6] and metastatic disease $[5,15]$.

The mechanisms underlying the up-regulation of p130Cas/BCAR1 and Nedd9 in breast cancer are still largely unknown. However, as a concomitant increase of protein and mRNA levels has been reported [3,6], alterations in their transcription and/or mRNA stability may contribute to deregulating the expression of these two genes in mammary tumours. To date, several signalling pathways relevant to breast cancer aetiology, including transforming growth factor (TGF)-beta, progesterone and hypoxia signalling, have been found to positively regulate Nedd9 mRNA expression [2], but less is known about the transcriptional control of p130Cas/BCAR1. In breast cancer cells, $B C A R 1$ transcription is directly activated by the early growth response protein 1 (EGR1) in response to phorbol esters, but the presence of putative binding sites for additional transcription factors, such as NF-kB, p53 and HIF, in the promoter region [16] points to a more complex control of $B C A R 1$ gene expression.

In the absence of an oncogenic hit, over-expression of either p130Cas/BCAR1 or Nedd9 is not sufficient to trigger mammary cell transformation. Nevertheless, up-regulation of these two Cas proteins seems to represent a convenient means for breast cancer cells to concomitantly boost multiple signalling pathways useful for tumour growth and invasion. Alternatively, increased Cas levels skew signalling cascades towards the preferential activation of specific downstream effectors.

For instance, high levels of p130Cas/BCAR1 in mammary epithelial cells subvert the function of TGF-beta from a negative regulator of tumour formation to a promoter of growth and dissemination. Elevated expression of p130Cas/BCAR1 diminishes the ability of TGF-beta to activate Smad2/3, but strengthens its coupling to p38 mitogen-activated protein kinase (p38 MAPK), thus rendering mammary cells resistant to TGF-beta-induced growth arrest. On the other hand, depletion of p130Cas/ BCAR1 expression in transformed mammary cells increases the activation of $\mathrm{Smad} 2 / 3$ and suppresses their ability to invade in response to TGF-beta [9].

The involvement of p130Cas/BCAR1 in numerous pathways driving mammary cell transformation is due, at least in part, to its function as a mediator of integrindependent signalling. Adhesion to the extracellular matrix induces p130Cas/BCAR1 phosphorylation and association with Src and focal adhesion kinase (FAK), which in turn promote survival signalling and activation of the cellular migration machinery $[1,2]$.
Disruption of the interaction between p130Cas/BCAR1 and FAK is sufficient to inhibit mammary tumourigenesis driven by the polyoma middle $\mathrm{T}$ oncogene (PyMT) [17]. Moreover, activating mutations in the Ras or phosphoinositide 3-kinase (PI3K) pathway do not overcome the requirement of a functional p130Cas/FAK signalling complex for the survival and growth of human breast cancer cells [17]. A recent study shows not only that p130Cas/BCAR1 is necessary for Src-dependent mammary cell transformation but also that the interplay between p130Cas/BCAR1 and Src is strictly controlled by the E3 ubiquitin-ligase Cullin 5 (Cul5). Src-mediated phosphorylation of p130Cas/ BCAR1 at specific tyrosine residues is responsible for Cas binding to the Cul5 adaptor SOCS6 and its consequent ubiquitylation by Cul5. Notably, loss of Cul5 results in p130Cas protein stabilization and facilitates the transformation of mammary epithelial cells by Src [18].

The ability of p130Cas/BCAR1 to control the remodelling of the actin cytoskeleton represents a mechanism commonly used to induce migration of breast cancer cells. For example, the enzyme lysyl oxidase (LOX) confers a motile phenotype on breast cancer cells by facilitating the formation of a p130Cas/Crk/DOCK180 complex, which sustains Rac activity and subsequent actin polymerization [19]. Conversely, the LOX pro-peptide (LOX-PP), which is released during the maturation of the pro-LOX enzyme, reduces breast cancer cell motility by attenuating phosphorylation and activation of p130Cas/BCAR1 [20].

Additional mechanisms that implicate p130Cas/BCAR1 in breast cancer invasion have been described. Tumour invasiveness often requires epithelial-mesenchymal transition, during which cells lose adhesions to their neighbours and become more motile. The link between p130Cas/ BCAR1 and epithelial-mesenchymal transition is supported by the role of p130Cas/BCAR1 in the control of breast cancer cell plasticity. In fact, in the aggressive A17 mouse mammary tumour cells, p130Cas/BCAR1 silencing induces loss of mesenchymal features and acquirement of epithelial-like traits, including the re-expression of the cell-cell adhesion molecule E-cadherin. The mechanism whereby p130Cas/BCAR1 determines a mesenchymal and invasive phenotype resides in its capacity to positively regulate the expression of cyclooxygenase- 2 in a Src- and Jnk-dependent manner [21]. Furthermore, in human mammary epithelial cells both p130Cas/BCAR1 and Nedd9 promote, via Src, removal of E-cadherin from the plasma membrane and its lysosomal degradation [22].

The first direct in vivo evidence for a role of Nedd9 in mammary tumourigenesis comes from the study of the effects of NEDD9 gene ablation in the MMTV-PyMT mouse model [11]. This study revealed that depletion of Nedd9 impairs mammary tumour development by limiting the activation of multiple pro-oncogenic signalling proteins, including its binding partners Fak and Src as 
well as Ras downstream effectors. Specifically, due to a severe down-regulation of FAK activation, lack of Nedd9 results in defective cell spreading and migration and a greater susceptibility to anoikis [11].

Beyond its function as a mediator of a variety of integrindependent processes, such as cell adhesion, survival and migration, Nedd9 can further impact on mammary tumour cell behaviour through its ability to directly activate the mitotic regulatory kinase Aurora-A (AurA) [23]. In MCF-7 mammary tumour cells, up-regulation of Nedd9 results in enhanced AurA activity leading to centrosomal and mitotic spindle abnormalities, whilst a weaker effect can be seen after changes of p130Cas/BCAR1 expression [23].

In human breast cancer AurA expression is considered an independent marker of poor prognosis [24] and it has been recently found that not only does it positively correlate with the expression of Nedd9 but also that coexpression of these two proteins has a significantly higher prognostic value [5]. Whilst most invasive mammary carcinomas highly express AurA, few have AurA gene amplification, implying that post-transcriptional mechanisms may be responsible for the alterations in AurA protein levels. Interestingly, binding of Nedd9 to AurA protects AurA from degradation by blocking its association with the APC/C ubiquitin ligase complex. As a consequence, Nedd9 overexpression in breast cancer cells leads to AurA protein stabilization and decreases the efficacy of AurA inhibitors [5] (Figure 2). In addition to its role in regulating the mitotic cell machinery, Nedd9 cooperates with AurA to control actin cytoskeleton dynamics and motility of breast cancer cells. Nedd9, through activation of AurA, negatively regulates cortactin (CTTN) acetylation and favours its binding to F-actin, thus sustaining cell migration and invasion [25] (Figure 2).

Increased levels of Nedd9 protein significantly correlate with the transition from in situ to invasive mammary carcinoma [15]. This observation is in line with a novel function ascribed to Nedd9 at the invasive pseudopodia of breast cancer cells. Nedd9 is able to direct local matrix degradation by reducing the levels of TIMP2 on the cell surface and favouring the activation of MMP14, MMP2 and MMP9 matrix metalloproteinases [15]. Nedd9 has also been identified as one of the key genes that mediate the switch from cohesive to single cell motility induced by TGF-beta during breast cancer cell dissemination. Upregulation of Nedd9 by TGF-beta is indeed required for proper actin rearrangements at the leading edge and efficient amoeboid motility of migrating tumour cells [26]. Given the increasing evidence that depletion of Nedd9 drastically reduces migration and invasion of highly metastatic mammary tumour cells $[5,15,25]$, inhibition of Nedd9 expression and/or activity represents an attractive strategy to target aggressive breast cancer cells. However, the complex scenario resulting from Nedd9 depletion should be taken into account. Indeed, silencing of Nedd9 can also cause centrosomal and spindle defects because of an abnormal AurA kinase activation [23]. As well, cells from MMTV-PyMT; NEDD9-null mammary tumours

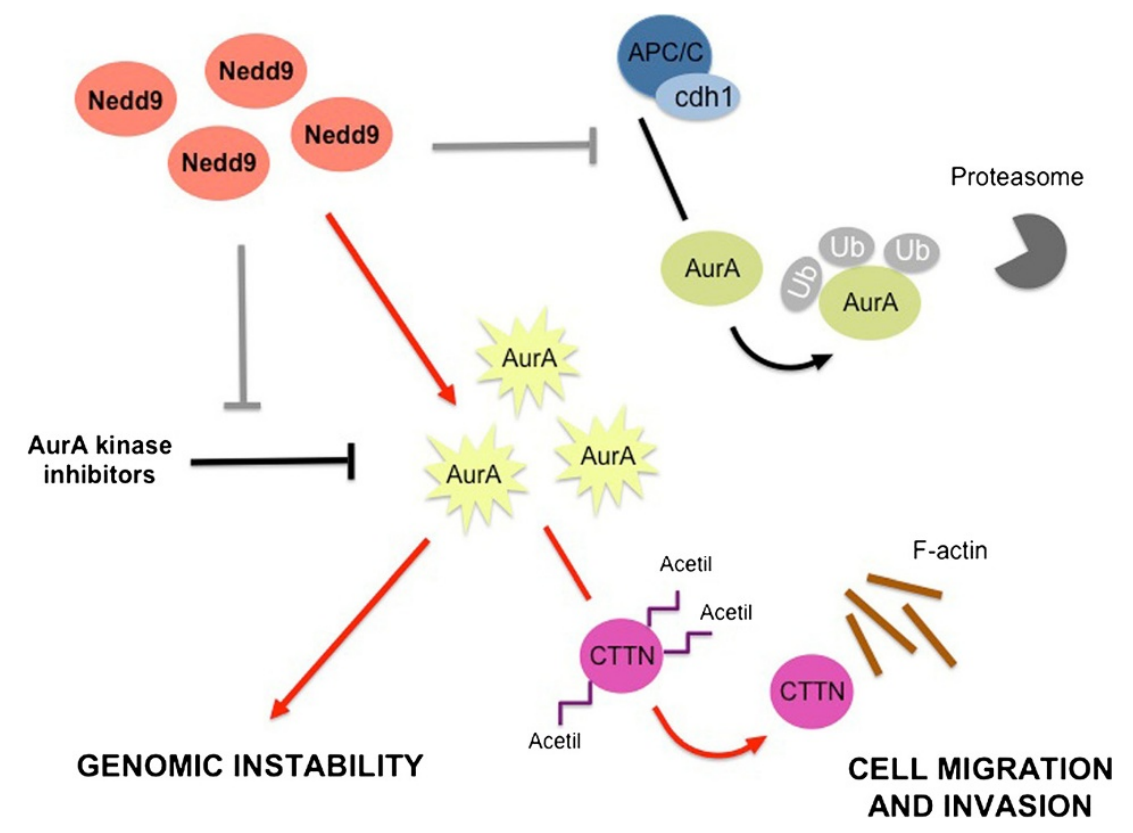

Figure 2 Schematic representation of the mechanisms of Aurora-A kinase regulation by Nedd 9 and major effects of Nedd 9 up-regulation mediated by Aurora-A kinase in breast cancer cells. AurA, Aurora-A; CTTN, cortactin; Ub, ubiquitin. 
exhibit pronounced genomic instability, which ultimately facilitates the selection of cells with a more aggressive phenotype [27].

The contribution of p130Cas/BCAR1 and Nedd9 to specific oncogenic pathways in different breast cancer subtypes is described in detail in the paragraphs below.

\section{p130Cas/BCAR1 and Nedd9 in estrogen receptor-positive breast cancer}

Expression of the oestrogen receptor (ER) in breast cancer inversely correlates with poor prognosis. Development and growth of ER-positive tumours are strictly dependent on the activity of ER, which, in addition to its well-described genomic action as a regulator of transcription, is also able to associate with cytoplasmic transducer molecules and activate non-genomic intracellular signalling cascades [28].

In ER-positive breast cancer, high levels of p130Cas/ BCAR1 are associated with poor prognosis and a higher risk of developing resistance to anti-oestrogen therapy $[13,29]$. The increased aggressiveness observed for ERpositive breast cancer expressing high p130Cas/BCAR1 levels may arise from the p130Cas/BCAR1-dependent activation of non-genomic ER signalling. Indeed, high levels of p130Cas/BCAR1 in T47D breast cancer cells promote the assembly of a multi-protein complex including ER $\alpha$, c-Src, and PI3K. In the presence of oestrogen, this macromolecular complex induces non-genomic ER proliferative signalling through Src and MAPK activation and the expression of cyclin D1. Conversely, silencing of endogenous p130Cas/BCAR1 is sufficient to markedly reduce oestrogen-dependent activation of Src and MAPKs as well as induction of cyclin D1 [30]. In parallel, in MCF-7 breast cancer cells, exposure to the selective ER modulator tamoxifen has been found to induce Src-mediated phosphorylation of p130Cas/BCAR1, which in turn promotes the activation of a pro-survival signalling pathway involving FAK and Akt [31] (Figure 3A).

At present, few data regarding a specific role for Nedd9 in ER-positive breast cancer are available, although, interestingly, its phosphorylation status has been linked to responsiveness to oestrogens. Indeed, ER-positive breast cancer cells preferentially express a hypophosphorylated form of Nedd9 (105 kDa Nedd9) rather than the highly phosphorylated form (115 kDa Nedd9), which is known to mediate cell spreading and migration [2]. In MCF7 cells, oestrogen treatment stabilizes the $105 \mathrm{kDa}$ Nedd9 form and suppresses the ability of Nedd9 to induce cell spreading, thus suggesting that Nedd9 phosphorylation status might be an indicator of breast cancer cell motility in response to oestrogens [32]. However, the specific pathways that are activated following oestrogen-dependent changes of Nedd9 phosphorylation have not been defined yet (Figure 3A).

\section{p130Cas/BCAR1 and Nedd9 in ErbB2 breast cancer}

Overexpression of the epidermal growth factor receptor 2 (ErbB2) occurs in approximately $25 \%$ of all breast cancers and is correlated with disease progression, decreased survival and metastasis. Such a poor prognosis is a likely reflection of the biological effects of ErbB2 overexpression, including increased cellular proliferation, anti-apoptosis, cell invasiveness and promotion of angiogenesis [33].

A positive correlation between the expression of BCAR1/ p130Cas and ErbB2 has been found in human breast cancers and the co-expression of these two genes is associated with shorter overall survival and a higher risk of developing distant metastasis [34]. Moreover, concomitant expression of p130Cas and ErbB2 proteins is related to a higher proliferative index [3].

The role of p130Cas/BCAR1 as a mediator of ErbB2 signalling was initially demonstrated in breast cancer cell lines devoid of ErbB receptors, in which over-expression of ErbB2 was sufficient to induce cell migration by triggering the coupling between p130Cas/BCAR1 and Crk [35]. Further in vivo analyses have shown that in the MMTV-NeuT mouse model of ErbB2 tumourigenesis, over-expression of p130Cas/BCAR1 accelerates the onset of mammary tumours, which are characterized at the molecular level by increased activation of c-Src and Akt [3]. Consistently, silencing of p130Cas/BCAR1 is sufficient to impair growth of MMTV-NeuT spontaneous tumours [36]. Mechanistically, the synergistic action of p130Cas/BCAR1 and ErbB2 is due to the ability of p130Cas/BCAR1 to interact with Erb2 and mediate the assembly of a functional molecular complex consisting of ErbB2, c-Src, and FAK [36].

p130Cas/BCAR1 cooperates with ErbB2 not only in mammary cell transformation but also in driving breast cancer cell migration and invasion and formation of metastasis [34,36,37]. A recent in vivo study indicates the interaction between p130Cas/BCAR1 and the FAKrelated kinase Pyk2 is required for ErbB2-transformed cells to invade and form metastasis [38]. Moreover, in MCF10A.B2 cells p130Cas/BCAR1 over-expression upon activation of ErbB2 induces cell invasion by strengthening ErbB2 downstream signalling and leading to enhanced Rac1 activation and MMP9 secretion [34,36]. The invasive phenotype resulting from the concomitant p130Cas/ BCAR1 over-expression and ErbB2 activation relies on a specific transcriptional program, which includes proteincoding genes and microRNAs involved in the control of amino acid synthesis, cell motility, migration, and angiogenesis [37] (Figure 3B).

The existence of mutual regulation between Nedd9 and ErbB2 is supported by independent studies showing that Nedd9 up-regulation increases the levels of ErbB2 mRNA [39] and, on the other hand, stimulation with the ErbB2 


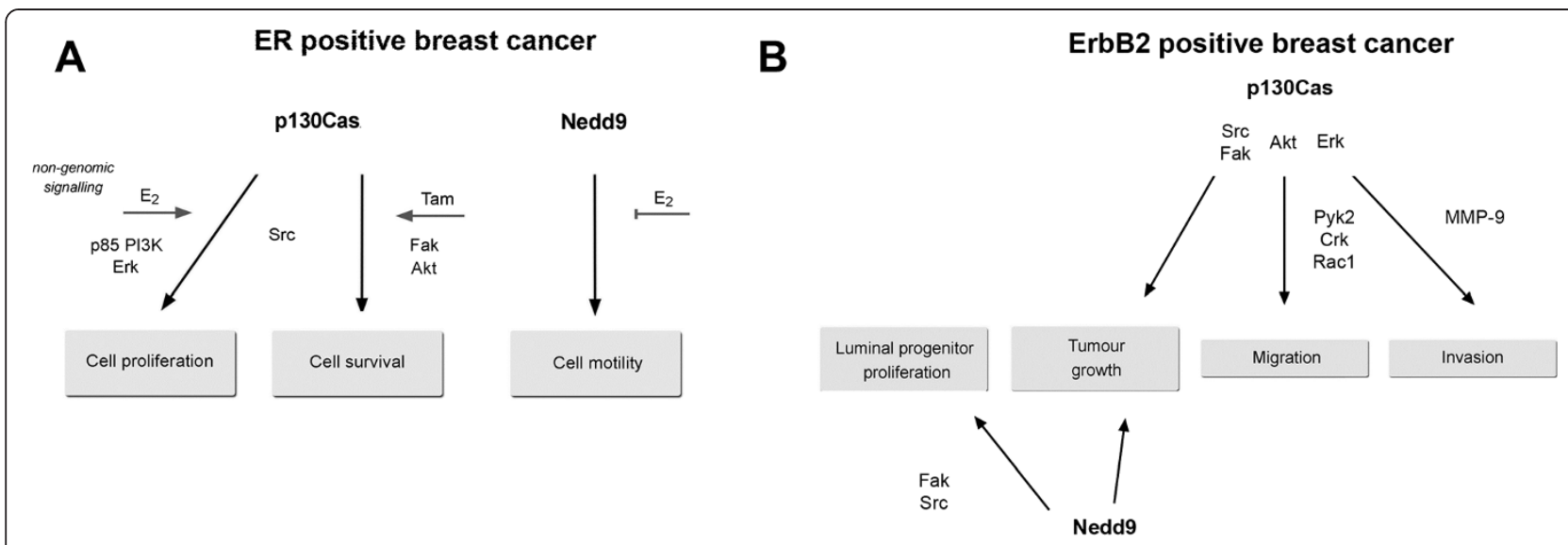

Triple negative breast cancer

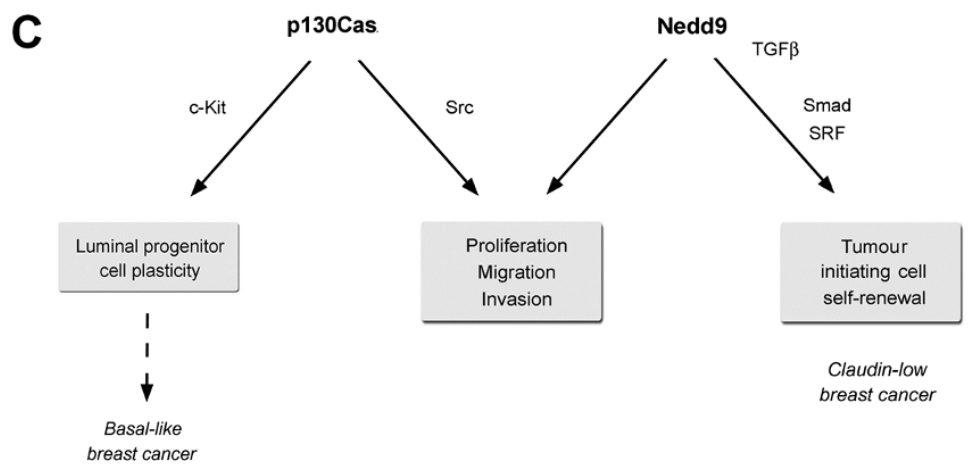

Figure 3 Biological effects and signalling pathways involving p130Cas/BCAR1 and Nedd9 in different breast cancer subtypes.

(A) Estrogen receptor (ER)-positive breast cancer. (B) ErbB2-positive breast cancer. (C) Triple-negative breast cancer. PI3K, phosphoinositide 3-kinase; SRF, serum response factor; TGF, transforming growth factor.

receptor ligand heregulin induces Nedd9 expression $[40,41]$. The evidence of a functional role for Nedd9 in ErbB2-dependent tumourigenesis has been provided by the analysis of MMTV-ErbB2 mice with the genetic deletion of NEDD9, demonstrating that MMTV-ErbB2; Nedd9-null mice are strongly resistant to tumour formation. The requirement of Nedd9 expression at the early stages of ErbB2-dependent tumourigenesis reflects its role in the control of pre-tumourous luminal progenitor cell growth. Specifically, in the absence of Nedd9, MMTV-ErbB2-luminal progenitors exhibit profound defects in the activation of adhesion signalling pathways together with a markedly reduced proliferative potential [12].

Since loss of either Nedd9 or p130Cas/BCAR1 impairs growth of ErbB2 tumours, the full activation of crucial ErbB2 downstream effectors, such as Src and FAK, may require the co-expression of Nedd9 and p130Cas/BCAR1, suggesting that these two Cas proteins cooperate during early ErbB2 tumourigenesis (Figure 3B).

\section{p130Cas/BCAR1 and Nedd9 in triple-negative breast cancer}

Triple-negative breast cancer (TNBC) is an aggressive breast cancer subtype defined by the lack of ER, progesterone receptor and human epidermal growth factor receptor 2 (ErbB2). Despite these distinctive histological features, TNBC comprises a highly heterogeneous group and encompasses a number of intrinsic molecular subtypes, most frequently basal-like and claudin-low [42].

Increasing data indicate that basal-like breast cancer originates from mammary luminal progenitors [43]. p130Cas/BCAR1 up-regulation is sufficient to confer basal cell features on luminal progenitors and to impair their full maturation in response to lactogenic stimuli. These defects in luminal progenitor differentiation together with the deregulation of the c-Kit receptor, which result from p130Cas/BCAR1 over-expression, closely resemble the phenotypes associated with Brcal deficiency in the mammary epithelium [43]. Together, these observations indicate that p130Cas/BCAR1 up-regulation might 
be a priming event in the development of basal-like breast cancer. Consistent with this, p130Cas/BCAR1 has been found commonly over-expressed in human TNBC [4].

Phosphoproteomic data from human breast cancer cell lines have revealed that basal cancer cells exhibit higher tyrosine phosphorylation of p130Cas/BCAR1 compared with luminal cancer cells, despite similar p130Cas/BCAR1 expression in the two subgroups [44]. In line with this, tyrosine phosphorylation of the p130Cas/BCAR1 SD has emerged not only as a distinguishing feature of ER-negative breast cancer cell lines compared with ERpositive lines, but also crucial for the proliferation, migration and invasion of these cells [45] (Figure 3C).

Analysis of Nedd9 expression across different breast cancers has revealed a correlation of this gene with the TNBC subtype. TNBC cells require Nedd9 expression to maintain their mesenchymal phenotype and to migrate and invade in vitro [6]. Moreover, in contrast to ER-positive cells, ER-negative breast cancer cells predominantly express a hyper-phosphorylated form of Nedd9 (115 kDa Nedd9), which represents the pool of Nedd9 able to sustain cell adhesion and migration signalling [32].

Lastly, Nedd9 has been found over-expressed in claudin low breast cancers compared with other molecular subtypes. In claudin low breast cancer cell lines TGF-beta up-regulates Nedd9 expression by inducing Smad and MRTF-SRF protein binding to regulatory regions within the NEDD9 locus. Nedd9 induction is necessary to establish a positive feedback loop that integrates TGF-beta/ Smad and Rho-actin-SRF signalling and drives the expansion of tumour-initiating cells, selectively in claudin low breast cancer, upon TGF-beta stimulation [7] (Figure 3C).

\section{p130Cas/BCAR1 and Nedd9 in anti-oestrogen resistance}

Tamoxifen is the most commonly used anti-oestrogen in the treatment of breast cancer. Patients with ER-positive breast tumours may initially benefit from this treatment, but a significant proportion of these initially responding patients acquire resistance to tamoxifen over time and the disease progresses [46].

p130Cas/BCAR1 has been identified as a gene responsible for the resistance to the anti-proliferative effects of tamoxifen [29,47]. In general, in ER-positive breast cancer patients, high levels of p130Cas/BCAR1 correlate with earlier relapse and shorter overall survival [14].

A first hint as to the possible mechanisms underlying the up-regulation of p130Cas/BCAR1 in tamoxifen-resistant cells comes from the analysis of the BCAR1 gene promoter. This study shows that $B C A R 1$ gene transcription is positively regulated by the transcription factor EGR1 and its co-regulator NAB2. Importantly, the increased expression of NAB2 and the enhanced EGR1 binding to the $B C A R 1$ promoter observed in tamoxifen-resistant cells is likely to be responsible for the constitutive high expression of p130Cas/BCAR1 [16].

Several mechanisms have been proposed to explain the correlation between p130Cas/BCAR1 expression and resistance to tamoxifen. A major determinant of the p130Cas/BCAR1-dependent increased proliferation of tamoxifen-resistant cells is the tyrosine phosphorylation of the p130Cas/BCAR1 SD. Indeed, tyrosine phosphorylation of the p130Cas/BCAR1 SD is induced by tamoxifen and correlates with tamoxifen-induced ER antagonist effects [31]. Either silencing of p130Cas/BCAR1 or abrogation of p130Cas/BCAR1 signalling, by expressing a dominant-negative tyrosine phosphorylated SD, is sufficient to re-establish sensitivity to tamoxifen in resistant cells. Inhibition of p130Cas attenuates the activation of Erk1/2 and PI3K/Akt pathways and results in cell death in response to tamoxifen [48].

Resistance to anti-oestrogen therapy specifically depends on the substrate domain of p130Cas/BCAR1 and not of Nedd9. Indeed, studies performed using chimeric proteins containing defined domains of Nedd9 and p130Cas/BCAR1 indicate that differences in their substrate domains are responsible for the differential effect on anti-oestrogen resistance [49]. As the substrate domains of these two Cas proteins differ in number, spacing and sequence context of tyrosine motifs, a distinctive pattern of p130Cas/BCAR1 $\mathrm{SD}$ tyrosine phosphorylation may be responsible for the recruitment of specific $\mathrm{SH} 2$ domain-containing proteins and the subsequent assembly of molecular complexes driving anti-oestrogen-resistant proliferation.

An additional mechanism implicated in tamoxifen resistance relies on the interaction between p130Cas/ BCAR1 and the SH2-containing protein AND-34/ BCAR3. Although there is a clear correlation between




the emergence of tamoxifen resistance in breast cancer and the association of BCAR3 with p130Cas/BCAR1, the available data on the functional meaning of this interaction appear controversial [50]. However, it has been recently demonstrated that the binding of BCAR3 to p130Cas/BCAR1 increases the levels of phosphorylated p130Cas/BCAR1, ultimately potentiating Erk1/2 phosphorylation and sustaining BCAR1-dependent anti-oestrogen resistance [51] (Figure 4).

\section{Conclusion}

p130Cas/BCAR1 and Nedd9, two members of the Cas protein family, play a key role at multiple steps of mammary tumourigenesis. Abnormal expression and/or phosphorylation of these two scaffold proteins leads to the disruption of regulatory signalling circuits controlling mammary cell survival, differentiation, proliferation and migration.

Noteworthy, in spite of their structural similarity, p130Cas/BCAR1 and Nedd9 exert both redundant and specific functions in breast cancer cells. Although the functional role of these two proteins has been extensively studied, further investigations are required to fully understand the molecular mechanisms underlying the deregulation of their expression in cancer. Moreover, high-resolution mapping of the interactions between Cas proteins and their pro-growth/pro-migratory binding partners may provide a rationale for the design of novel targeted therapies. Nevertheless, the use of Cas proteins as biomarkers for cancer prognosis and drug responsiveness may improve the clinical management of breast cancer.

\section{Abbreviations}

ER: Oestrogen receptor; FAK: Focal adhesion kinase; MAPK: Mitogen-activated protein kinase; PI3K: Phosphoinositide 3-kinase; SD: Substrate domain: TGF: Transforming growth factor; TNBC: Triple-negative breast cancer.

\section{Competing interests}

The authors declare that they have no competing interests.

\section{Acknowledgements}

This work was supported by AIRC (Associazione Italiana Ricerca Cancro) to SC (IG11346) and PD (IG-11896); MIUR (FIRB giovani 2008 RBFR08F2FS to SC), MIUR (Ministero Università Ricerca, PRIN 2010/2011) to PD and Compagnia San Paolo, Torino; Progetto d'Ateneo, Università di Torino 2011 to PD. GT is supported by Breast Cancer Campaign UK (2012MayPR076).

\section{Author details}

${ }^{1}$ Department of Molecular Biotechnology and Health Sciences, University of Torino, Torino 10126, Italy. ${ }^{2}$ European Cancer Stem Cell Research Institute and Cardiff School of Biosciences, Cardiff University, Cardiff CF24 4HQ, UK.

\section{Published online: 25 September 2014}

\section{References}

1. Cabodi S, del Pilar C-LM, Di Stefano P, Defilippi P: Integrin signalling adaptors: not only figurants in the cancer story. Nat Rev Cancer 2010, 10:858-870

2. Tikhmyanova N, Little JL, Golemis EA: CAS proteins in normal and pathological cell growth control. Cell Mol Life Sci 2010, 67:1025-1048.
3. Cabodi S, Tinnirello A, Di Stefano P, Bisarò B, Ambrosino E, Castellano I, Sapino A, Arisio R, Cavallo F, Forni G, Glukhova M, Silengo L, Altruda F, Turco E, Tarone G, Defilippi P: p130Cas as a new regulator of mammary epithelial cell proliferation, survival, and HER2-neu oncogene-dependent breast tumorigenesis. Cancer Res 2006, 66:4672-4680.

4. Tornillo G, Elia AR, Castellano I, Spadaro M, Bernabei P, Bisaro B, Camacho-Leal Mdel P, Pincini A, Provero P, Sapino A, Turco E, Defilippi P, Cabodi S: p130Cas alters the differentiation potential of mammary luminal progenitors by deregulating c-Kit activity. Stem Cells 2013, 31:1422-1433.

5. Ice RJ, McLaughlin SL, Livengood RH, Culp MV, Eddy ER, Ivanov AV, Pugacheva EN: NEDD9 depletion destabilizes Aurora A kinase and heightens the efficacy of Aurora A inhibitors: implications for treatment of metastatic solid tumors. Cancer Res 2013, 73:3168-3180.

6. Kong C, Wang C, Wang L, Ma M, Niu C, Sun X, Du J, Dong Z, Zhu S, Lu J, Huang B: NEDD9 is a positive regulator of epithelial-mesenchymal transition and promotes invasion in aggressive breast cancer. PLoS One 2011, 6:e22666.

7. Bruna A, Greenwood W, Le Quesne J, Teschendorff A, Miranda-Saavedra D, Rueda OM, Sandoval JL, Vidakovic AT, Saadi A, Pharoah P, Stingl J, Caldas C: TGFbeta induces the formation of tumour-initiating cells in claudinlow breast cancer. Nat Commun 2012, 3:1055.

8. Camacho Leal Mdel P, Pincini A, Tornillo G, Fiorito E, Bisaro B, Di Luca E, Turco E, Defilippi P, Cabodi S: p130Cas over-expression impairs mammary branching morphogenesis in response to estrogen and EGF. PLOS One 2012, 7:e49817.

9. Wendt MK, Smith JA, Schiemann WP: p130Cas is required for mammary tumor growth and transforming growth factor-beta-mediated metastasis through regulation of Smad2/3 activity. J Biol Chem 2009, 284:34145-34156.

10. Zhao Y, Kumbrink J, Lin BT, Bouton AH, Yang S, Toselli PA, Kirsch KH: Expression of a phosphorylated substrate domain of p130Cas promotes PyMT-induced c-Src-dependent murine breast cancer progression. Carcinogenesis 2013, 34:2880-2890.

11. Izumchenko E, Singh MK, Plotnikova OV, Tikhmyanova N, Little $J$, Serebriiskii IG, Seo S, Kurokawa M, Egleston BL, Klein-Szanto A, Pugacheva EN, Hardy RR, Wolfson M, Connolly DC, Golemis EA: NEDD9 promotes oncogenic signaling in mammary tumor development. Cancer Res 2009, 69:7198-7206

12. Little JL, Serzhanova V, Izumchenko E, Egleston BL, Parise E, Klein-Szanto AJ, Loudon G, Shubina M, Seo S, Kurokawa M, Ochs MF, Golemis EA: A requirement for Nedd9 in luminal progenitor cells prior to mammary tumorigenesis in MMTV-HER2/ErbB2 mice. Oncogene 2014, 33:411-420.

13. van der Flier S, Chan CM, Brinkman A, Smid M, Johnston SR, Dorssers LC, Dowsett M: BCAR1/p130Cas expression in untreated and acquired tamoxifen-resistant human breast carcinomas. Int J Cancer 2000, 89:465-468.

14. Dorssers LC, Grebenchtchikov N, Brinkman A, Look MP, van Broekhoven SP, de Jong D, Peters HA, Portengen H, Meijer-van Gelder ME, Klijn JG, van Tienoven DT, Geurts-Moespot A, Span PN, Foekens JA, Sweep FC: The prognostic value of BCAR1 in patients with primary breast cancer. Clin Cancer Res 2004, 10:6194-6202.

15. McLaughlin SL, Ice RJ, Rajulapati A, Kozyulina PY, Livengood RH, Kozyreva VK, Loskutov YV, Culp MV, Weed SA, Ivanov AV, Pugacheva EN: NEDD9 depletion leads to MMP14 inactivation by TIMP2 and prevents invasion and metastasis. Mol Cancer Res 2014, 12:69-81.

16. Kumbrink J, Kirsch KH: Regulation of p130(Cas)/BCAR1 expression in tamoxifen-sensitive and tamoxifen-resistant breast cancer cells by EGR1 and NAB2. Neoplasia 2012, 14:108-120.

17. Pylayeva Y, Gillen KM, Gerald W, Beggs HE, Reichardt LF, Giancotti FG: Ras- and PI3K-dependent breast tumorigenesis in mice and humans requires focal adhesion kinase signaling. J Clin Invest 2009, 119:252-266.

18. Teckchandani A, Laszlo GS, Simo S, Shah K, Pilling C, Strait AA, Cooper JA: Cullin5 destabilizes Cas to inhibit Src-dependent cell transformation. J Cell Sci 2014, 127:509-520.

19. Payne SL, Hendrix MJ, Kirschmann DA: Lysyl oxidase regulates actin filament formation through the p130(Cas)/Crk/DOCK180 signaling complex. J Cell Biochem 2006, 98:827-837.

20. Zhao Y, Min C, Vora SR, Trackman PC, Sonenshein GE, Kirsch KH: The lysyl oxidase pro-peptide attenuates fibronectin-mediated activation of focal 
adhesion kinase and p130Cas in breast cancer cells. J Biol Chem 2009, 284:1385-1393.

21. Bisaro B, Montani M, Konstantinidou G, Marchini C, Pietrella L, lezzi M, Galiè M, Orso F, Camporeale A, Colombo SM, Di Stefano P, Tornillo G, Camacho-Leal MP, Turco E, Taverna D, Cabodi S, Amici A, Defilippi P: p130Cas/Cyclooxygenase-2 axis in the control of mesenchymal plasticity of breast cancer cells. Breast Cancer Res 2012, 14:R137.

22. Tikhmyanova N, Golemis EA: NEDD9 and BCAR1 negatively regulate E-cadherin membrane localization, and promote E-cadherin degradation. PLoS One 2011, 6:e22102

23. Pugacheva EN, Golemis EA: The focal adhesion scaffolding protein HEF1 regulates activation of the Aurora-A and Nek2 kinases at the centrosome. Nat Cell Biol 2005, 7:937-946.

24. Nadler Y, Camp RL, Schwartz C, Rimm DL, Kluger HM, Kluger Y: Expression of Aurora A (but not Aurora B) is predictive of survival in breast cancer. Clin Cancer Res 2008, 14:4455-4462.

25. Kozyreva VK, McLaughlin SL, Livengood RH, Calkins RA, Kelley LC, Rajulapati A, Ice RJ, Smolkin MB, Weed SA, Pugacheva EN: NEDD9 regulates actin dynamics through cortactin deacetylation in an AURKA/HDAC6dependent manner. Mol Cancer Res 2014, 12:681-693.

26. Giampieri S, Manning C, Hooper S, Jones L, Hill CS, Sahai E: Localized and reversible TGFbeta signalling switches breast cancer cells from cohesive to single cell motility. Nat Cell Biol 2009, 11:1287-1296.

27. Singh MK, Izumchenko E, Klein-Szanto AJ, Egleston BL, Wolfson M, Golemis EA: Enhanced genetic instability and dasatinib sensitivity in mammary tumor cells lacking NEDD9. Cancer Res 2010, 70:8907-8916.

28. Silva CM, Shupnik MA: Integration of steroid and growth factor pathways in breast cancer: focus on signal transducers and activators of transcription and their potential role in resistance. Mol Endocrinol 2007, 21:1499-1512

29. Brinkman A, van der Flier S, Kok EM, Dorssers LC: BCAR1, a human homologue of the adapter protein p130Cas, and antiestrogen resistance in breast cancer cells. J Natl Cancer Inst 2000, 92:112-120.

30. Cabodi S, Moro L, Baj G, Smeriglio M, Di Stefano P, Gippone S, Surico N, Silengo L, Turco E, Tarone G, Defilippi P: p130Cas interacts with estrogen receptor alpha and modulates non-genomic estrogen signaling in breast cancer cells. J Cell Sci 2004, 117:1603-1611.

31. Cowell LN, Graham JD, Bouton AH, Clarke CL, O'Neill GM: Tamoxifen treatment promotes phosphorylation of the adhesion molecules, p130Cas/BCAR1, FAK and Src, via an adhesion-dependent pathway. Oncogene 2006, 25:7597-7607.

32. Bradshaw LN, Zhong J, Bradbury P, Mahmassani M, Smith JL, Ammit AJ, O'Neill GM: Estradiol stabilizes the 105-kDa phospho-form of the adhesion docking protein NEDD9 and suppresses NEDD9-dependent cell spreading in breast cancer cells. Biochim Biophys Acta 1813, 2011:340-345.

33. Moasser MM: The oncogene HER2: its signaling and transforming functions and its role in human cancer pathogenesis. Oncogene 2007, 26:6469-6487

34. Tornillo G, Bisaro B, Camacho-Leal Mdel P, Galie M, Provero P, Di Stefano P, Turco E, Defilippi P, Cabodi S: p130Cas promotes invasiveness of three-dimensional ErbB2-transformed mammary acinar structures by enhanced activation of mTOR/p70S6K and Rac1. Eur J Cell Biol 2011, 90:237-248.

35. Spencer KS, Graus-Porta D, Leng J, Hynes NE, Klemke RL: ErbB2 is necessary for induction of carcinoma cell invasion by ErbB family receptor tyrosine kinases. J Cell Biol 2000, 148:385-397.

36. Cabodi S, Tinnirello A, Bisaro B, Tornillo G, del Pilar Camacho-Leal M, Forni G, Cojoca R, lezzi M, Amici A, Montani M, Eva A, Di Stefano P, Muthuswamy SK, Tarone G, Turco E, Defilippi P: p130Cas is an essential transducer element in ErbB2 transformation. FASEB J 2010, 24:3796-3808.

37. Pincini A, Tornillo G, Orso F, Sciortino M, Bisaro B, Leal Mdel P, Lembo A, Brizzi MF, Turco E, De Pittà C, Provero P, Medico E, Defilippi P, Taverna D, Cabodi S: Identification of p130Cas/ErbB2-dependent invasive signatures in transformed mammary epithelial cells. Cell Cycle 2013, 12:2409-2422.

38. Lahlou H, Sanguin-Gendreau V, Frame MC, Muller WJ: Focal adhesion kinase contributes to proliferative potential of ErbB2 mammary tumour cells but is dispensable for ErbB2 mammary tumour induction in vivo. Breast Cancer Res 2012, 14:R36.

39. Fashena SJ, Einarson MB, O'Neill GM, Patriotis C, Golemis EA: Dissection of HEF1-dependent functions in motility and transcriptional regulation. J Cell Sci 2002, 115:99-111.
40. Nagashima T, Oyama M, Kozuka-Hata H, Yumoto N, Sakaki Y, Hatakeyama M: Phosphoproteome and transcriptome analyses of ErbB ligand-stimulated MCF-7 cells. Cancer Genomics Proteomics 2008, 5:161-168.

41. Worthington J, Bertani M, Chan HL, Gerrits B, Timms JF: Transcriptional profiling of ErbB signalling in mammary luminal epithelial cells - interplay of ErbB and IGF1 signalling through IGFBP3 regulation. BMC Cancer 2010, 10:490.

42. Metzger-Filho O, Tutt A, de Azambuja E, Saini KS, Viale G, Loi S, Bradbury I, Bliss JM, Azim HA Jr, Ellis P, Di Leo A, Baselga J, Sotiriou C, Piccart-Gebhart M: Dissecting the heterogeneity of triple-negative breast cancer. J Clin Oncol 2012, 30:1879-1887.

43. Molyneux G, Smalley MJ: The cell of origin of BRCA1 mutation-associated breast cancer: a cautionary tale of gene expression profiling. J Mammary Gland Biol Neoplasia 2011, 16:51-55.

44. Hochgräfe F, Zhang L, OToole SA, Browne BC, Pinese M, Porta Cubas A, Lehrbach GM, Croucher DR, Rickwood D, Boulghourjian A, Shearer R, Nair R, Swarbrick A, Faratian D, Mullen P, Harrison DJ, Biankin AV, Sutherland RL, Raftery MJ, Daly RJ: Tyrosine phosphorylation profiling reveals the signaling network characteristics of basal breast cancer cells. Cancer Res 2010, 70:9391-9401.

45. Cunningham-Edmondson AC, Hanks SK: p130Cas substrate domain signaling promotes migration, invasion, and survival of estrogen receptor-negative breast cancer cells. Breast Cancer 2009, 1:39-52.

46. Dorssers LC, Van der Flier S, Brinkman A, van Agthoven T, Veldscholte J, Berns EM, Klijn JG, Beex LV, Foekens JA: Tamoxifen resistance in breast cancer: elucidating mechanisms. Drugs 2001, 61:1721-1733.

47. van Agthoven T, Veldscholte J, Smid M, van Agthoven TL, Vreede L, Broertjes M, de Vries I, de Jong D, Sarwari R, Dorssers LC: Functional identification of genes causing estrogen independence of human breast cancer cells. Breast Cancer Res Treat 2009, 114:23-30.

48. Soni S, Lin BT, August A, Nicholson RI, Kirsch KH: Expression of a phosphorylated p130(Cas) substrate domain attenuates the phosphatidylinositol 3-kinase/Akt survival pathway in tamoxifen resistant breast cancer cells. J Cell Biochem 2009, 107:364-375.

49. Brinkman A, de Jong D, Tuinman S, Azaouagh N, van Agthoven $T$, Dorssers LC: The substrate domain of BCAR1 is essential for anti-estrogen-resistant proliferation of human breast cancer cells. Breast Cancer Res Treat 2010, 120:401-408.

50. Near Rl, Zhang Y, Makkinje A, Vanden Borre P, Lerner A: AND-34/BCAR3 differs from other NSP homologs in induction of anti-estrogen resistance, cyclin D1 promoter activation and altered breast cancer cell morphology. J Cell Physiol 2007, 212:655-665.

51. Wallez Y, Riedl SJ, Pasquale EB: Association of the BCAR1 and BCAR3 scaffolding proteins in cell signaling and antiestrogen resistance. J Biol Chem 2014, 289:10431-10444.

\section{doi:10.1186/s13058-014-0443-5}

Cite this article as: Tornillo et al:: Cas proteins: dodgy scaffolding in breast cancer. Breast Cancer Research 2014 16:443. 